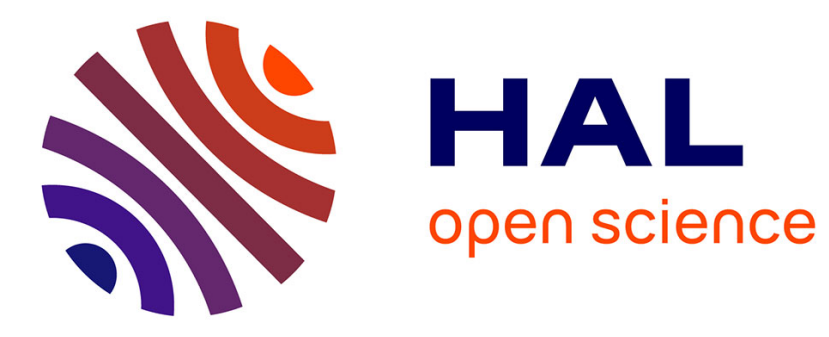

\title{
La fin des quotas laitiers, entre craintes et espoirs
}

Vincent Chatellier

\section{To cite this version:}

Vincent Chatellier. La fin des quotas laitiers, entre craintes et espoirs. Pour : enquêtes et témoignages, 2015, 225, pp.7-11. hal-02629818

\section{HAL Id: hal-02629818 \\ https://hal.inrae.fr/hal-02629818}

Submitted on 27 May 2020

HAL is a multi-disciplinary open access archive for the deposit and dissemination of scientific research documents, whether they are published or not. The documents may come from teaching and research institutions in France or abroad, or from public or private research centers.
L'archive ouverte pluridisciplinaire HAL, est destinée au dépôt et à la diffusion de documents scientifiques de niveau recherche, publiés ou non, émanant des établissements d'enseignement et de recherche français ou étrangers, des laboratoires publics ou privés. 


\section{actualités}

\section{agriculture}

\section{La fin des quotas laitiers, entre craintes et espoirs}

Depuis le $1^{\text {er }}$ avril 2015, date de la suppression des quotas laitiers, la filière laitière européenne est entrée dans une nouvelle phase de son histoire. Après trois décennies d'un contrôle strict de l'offre de lait à l'échelle de chaque État membre, les autorités communautaires ont décidé de mettre un terme à la politique de contingentement (Mahé et Guyomard, 1993). Ce choix s'inscrit dans la logique politique qui prévaut depuis plusieurs années et qui se caractérise par un désengagement progressif de la puissance publique dans la régulation des marchés agricoles linstauration du découplage des aides directes, baisse des restitutions aux exportations, conditions plus restrictives au déclenchement du stockage public, etc.). Les principaux arguments déployés par les tenants de la suppression des quotas laitiers, et qui font l'objet de controverses (Pflimlin, 2010), sont les suivants : les quotas ne favorisent pas une allocation optimale des ressources et contribuent à freiner le processus de concentration de la production dans les exploitations et les régions ayant des avantages comparatifs ; ils génèrent des rentes car l'entrée dans le secteur est conditionnée à l'obtention d'un droit à produire ; ils constituent un instrument du passé dans la mesure où ils étaient combinés à d'autres outils de la Politique Agricole Commune (PAC) qui ont eux-mêmes évolué ou disparu ; ils ne sont plus adaptés à la situation internationale actuelle en raison des opportunités de développement qu'offre, pour l'Union Européenne (UE), la croissance de la consommation dans les pays émergents (International Dairy Federation, 2014).

Après un rapide retour historique sur cette politique de contingentement et sur les choix français arrêtés pour sa mise en œuvre, une réflexion est proposée autour de deux questions principales : comment les quotas laitiers ont-ils influencé la dynamique du secteur laitier français ? Quelles sont les principales pistes d'évolution pour la période post-quota?

\section{Un bref retour historique sur une politique déjà ancienne}

Entre 1968, date de création de l'Organisation Commune de Marché (OCM) du lait et des produits laitiers, et 1984, la production laitière européenne a augmenté à un rythme soutenu. Les instruments de régulation mis en œuvre dans le cadre de la PAC et le développement de la consommation intérieure ont offert un cadre économique propice au développement de la production laitière. Celui-ci a également été favorisé par la modification des techniques (diffusion du modèle maïs fourrage soja), l'intensification des surfaces 
fourragères, le progrès de la génétique animale et l'amélioration des infrastructures dédiées à cette activité. Il en a résulté une croissance de l'offre plus rapide que celle de la consommation et, de facto, la constitution d'excédents de produits laitiers de plus en plus coûteux pour les contribuables lun tiers des dépenses de la PAC relevait à cette époque du secteur laitier). Suite à plusieurs tentatives infructueuses de régulation de l'offre, dont l'application d'une taxe de coresponsabilité, les autorités ont finalement opté pour l'application des quotas.

Lors de la mise en œuvre des quotas laitiers, le consensus n'était pas généralisé, tant entre les États membres qu'entre les différentes parties prenantes. De nombreux agriculteurs redoutaient, en effet, que l'application des quotas soit un frein au développement de leurs exploitations et nuisent à l'esprit d'entreprise. Certains négociateurs, dont ceux du Royaume-Uni, exprimaient une préférence pour une autre forme de régulation comme celle consistant à recourir à une baisse intense des prix garantis. Cette dernière option n'a finalement pas été retenue car le risque d'une baisse soudaine et drastique du nombre de producteurs était réel.

En France, les gouvernements successifs ont toujours veillé à utiliser les latitudes offertes par le règlement communautaire sur les quotas laitiers, d'une part, pour favoriser les exploitations familiales de dimension moyenne et, d'autre part, pour limiter le processus engagé de concentration de la production dans les régions bénéficiant d'avantages comparatifs (Butault et al., 1984). Ainsi, par exemple, les exploitations laitières localisées dans les zones de montagne ont bénéficié d'un traitement de faveur en n'étant pas soumises à la même réduction du quota que celles de plaine (Dervillé et Allaire, 2014). De même, un lien fort entre le quota et le foncier a été privilégié, selon une mécanique parfois complexe, de façon à ce que la production laitière demeure ancrée au territoire. La gestion administrative des quotas a été décentralisée à l'échelle de chaque département, du moins jusqu'en 2011, date de création des neuf bassins de production. Ces choix diffèrent de ceux arrêtés dans d'autres États membres, comme aux Pays-Bas, au RoyaumeUni ou en Allemagne, où un système de valorisation marchande des droits à produire a été privilégié (Barthélémy et David, 1999).

\section{Comment les quotas \\ ont-ils influencé la dynamique du secteur laitier français ?}

Les quotas laitiers ont permis de maîtriser les volumes européens de lait par l'application d'une pénalité dissuasive à l'échelle du producteur. Il en a résulté une baisse des stocks publics d'intervention et une diminution des dépenses correspondantes. En 2008, les autorités communautaires ont décidé d'augmenter le niveau du quota laitier européen de l'ordre de $1 \%$ par an sur la période 2009-2015 de façon à favoriser un atterrissage en douceur du dispositif. Ainsi, en France, le quota de production est devenu supérieur à la production laitière depuis déjà de nombreuses années.

Au plan territorial, les quotas laitiers ont contribué à maintenir une activité 
laitière dans de nombreuses petites régions agricoles françaises, y compris dans les zones de montagne où, en outre, des soutiens spécifiques du deuxième pilier de la PAC sont accordés lindemnités compensatoires de handicaps naturels, prime herbagère agro-environnementale, etc.). Avec $21 \%$ des exploitations laitières françaises en 2014 , les zones de montagne fournissent $14 \%$ de la collecte de lait, dans des conditions de rémunération du producteur qui varient cependant fortement selon le niveau de valorisation des produits finis (filières fromagères à valeur ajoutée ou non). Le mouvement de concentration géographique de la production, constaté avant la mise en œuvre des quotas, a été nettement freiné depuis 1984. Les trois régions de l'Ouest (Basse-Normandie, Bretagne et Pays-de-la-Loirel ne sont pas parvenues, tout au long des trois dernières décennies, à augmenter leur contribution relative à l'offre nationale de lait lqui varie entre $46 \%$ et $49 \%$ selon les années), ce malgré l'existence d'avantages comparatifs (densité de collecte, hauts rendements fourragers, proximité des ports, etc.). En dépit de la volonté politique de maintenir le lait dans les territoires, un recul significatif de la collecte de lait a été observé depuis 1984 dans certaines zones de polyculture-élevage à faible densité laitière : $-32 \%$ en Poitou-Charentes et en Aquitaine, -24\% en Midi-Pyrénées, $-22 \%$ en Bourgogne et $-20 \%$ dans la région Centre (contre - $8 \%$ en moyenne nationale).

Au niveau des exploitations, les quotas laitiers ont sûrement favorisé un rythme de restructuration plus lent que ce qui serait advenu avec une autre option de régulation. Ils n'ont cependant pas empêché une profonde baisse des effectifs en raison des gains de productivité exceptionnels observés au niveau du facteur travail. Ainsi, le nombre d'exploitations livrant du lait à l'industrie a été divisé par près de six en France pour atteindre 67000 en 2014 (Perrot et al., 2014). Ce recul est encore plus important (division par au moins dix des effectifs) dans les régions suivantes: Alsace, Aquitaine, Bourgogne, Centre et Poitou-Charentes. Les exploitations résiduelles assurent, en moyenne 2014, une production de $367000 \mathrm{~kg}$ de lait par an. Cependant, d'ores et déjà 8500 exploitations ont un quota supérieur à $600000 \mathrm{~kg}$ et $20 \%$ des vaches laitières sont localisées dans des étables de plus de 100 vaches laitières. Les formes sociétaires IGAEC, EARL, SARL et société civilel jouent aujourd'hui un rôle clé en fournissant près de $80 \%$ de la production laitière nationale.

\section{Quelles sont les principales pistes d'évolution pour la période post-quota?}

Dans un contexte où la demande de produits laitiers est en légère baisse sur le marché français et peu dynamique au sein de l'UE, l'évolution de la production laitière nationale dépendra d'abord de la compétitivité des entreprises à l'international (Jamet, 2014). La France a déjà démontré un certain savoir-faire dans ce domaine : elle occupe le troisième rang des pays européens exportateurs de produits laitiers derrière les Pays-Bas et l'Allemagne; sa balance commerciale (+ 3,5 milliards d'euros en 2014) a augmenté de 1,7 milliard d'euros depuis 
2000 sous l'impulsion surtout des pays tiers (+ 1,1 milliard d'euros). La concurrence reste néanmoins vive en raison de l'essor spectaculaire des exportations néo-zélandaises et, aussi, américaines (Chatellier et al., 2013). À long terme, les pays européens pourraient cependant profiter d'un ralentissement du rythme de croissance de l'offre dans ces deux zones concurrentes et de l'augmentation de la consommation dans les pays en développement.

En interne à l'UE, les jeux concurrentiels devraient s'accentuer, surtout entre l'Allemagne, le Danemark, la France, l'Irlande et les Pays-Bas. La compétition entre États membres n'était jusqu'alors que partielle dans la mesure où chaque pays disposait d'une protection de ses volumes au travers des quantités globales garanties. Désormais, les pays plus compétitifs seront en mesure de développer leur production au détriment des autres. Dans ce cadre, le secteur laitier français bénéficie de nombreux atouts : un climat favorable aux productions fourragères, élément indispensable pour optimiser les coûts de production; un prix du foncier plus bas que dans la plupart des pays concurrents; une intensification des surfaces plus modérée qu'ailleurs, ce qui offre parfois localement de réelles opportunités de développement (Lelyon et al., 2012) ; la présence de groupes industriels puissants et de plus en plus internationalisés.

La localisation géographique de la production laitière est également susceptible d'évoluer au sein de chaque État membre, en fonction surtout des différentiels de coûts de production entre zones, des opportunités ou non de diversification des activités agricoles (productions animales versus végétales), de l'impact des normes environnementales et des stratégies d'investissement des industriels. Un mouvement de concentration de la production dans les régions ayant déjà les plus fortes densités est attendu. II n'en demeure pas moins qu'une certaine diversité de modèles productifs devrait persister en France (Peyraud et Duhem, 2013), en raison de l'hétérogénéité des milieux naturels et des disponibilités foncières. Les modèles productifs hexagonaux recouvrent en effet des réalités bien différentes, avec des systèmes herbagers dans les zones de montagne; des systèmes intensifs et spécialisées à base de maïs ensilage et avec des superficies parfois modestes dans l'Ouest; des systèmes diversifiés avec une part conséquente de céréales dans les zones intermédiaires, etc. Une politique agricole volontariste au bénéfice des zones défavorisées restera essentielle du fait des écarts de productivité et des surcoûts induits. Dans ce sens, la réorientation des aides directes du premier pilier de la PAC, prévue en France au titre de la période 2015-2020, s'inscrit bien dans cette logique.

À compter de 2015, l'ajustement de l'offre de lait à la demande ne sera donc plus arbitré par des règles administratives strictes fixées par les pouvoirs publics, mais par les entreprises de la transformation au travers d'un système de contractualisation (You, 2015). Après trente années d'un interventionnisme public fort, en lien étroit avec le syndicalisme et l'interprofession, ce changement de cap n'est pas si simple à mettre en œuvre et exigera 
vraisemblablement quelques années de transition. Cela semble d'autant probable en France que les entreprises n'ont pas toutes les mêmes stratégies de développement (stabilité des approvisionnements ou essor des exportations sur les marchés tiers), ni les mêmes performances commerciales, ni la même manière de construire une relation contractuelle. Si la restructuration des exploitations devrait se poursuivre en raison surtout des améliorations technologiques (robotisation de la traite, automatisation de certaines tâches et spécialisation), la vitesse du processus n'est pas écrite et dépendra, pour partie, de choix sociétaux (consentement à payer des consommateurs pour des produits différenciés, prise en compte du bien-être animal, acceptabilité des élevages de très grande taille) et politiques (orientation des soutiens publics, limitation du processus de concentration par les normes).

\section{Vincent Chatellier}

Ingénieur de Recherche à l'INRA, UR 1134 (LERECO), Nantes

\section{Bibliographie}

Barthélémy D., David J., 1999. L'agriculture européenne et les droits à produire. Éditions INRA, $434 \mathrm{p}$.

Butault J.-P., Hairy D., Perraud D., 1984. «Crise laitière et quotas, remarques sur la régulation communautaire du marché du lait », Économie Rurale, n 163, p. 63-71.

Chatellier V., Lelyon B., Perrot C., You G., 2013. « Le secteur laitier français à la croisée des chemins », INRA Productions Animales, vol. 26 (2), p. 71-94.

Dervillé M., Allaire G., 2014. « Quelles perspectives pour les filières laitières de montagne après la suppression des quotas laitiers ? Une approche en termes de régime de concurrence », INRA Productions Animales, vol. 27 (1), p. 17-30.

International Dairy Federation, 2014. “The world dairy situation", Bulletin, n476, 230 p. Jamet J.-P., 2014. « Les défis de la sortie des quotas laitiers », Paysans, n³48, p. 11-18.

Lelyon B., Chatellier V., Daniel K., 2012. «Fin des quotas laitiers, contractualisation et stratégies productives : enseignements d'une modélisation bio économique », INRA Productions animales, 25 (1), p. 67-76.

Mahé L.P., Guyomard H., 1993. «Théorie de la production en présence de rationnements avec application. L'exemple des quotas laitiers dans la CEE », Revue Économique, vol 44 (1), p 71-94.

Perrot C., Caillaud D., Chatellier V., Ennifar M., You G., 2014. « La diversité des exploitations et des territoires laitiers français face à la fin des quotas », 21 ${ }^{\text {es }}$ Rencontres Recherches Ruminants (colloque 3R), Paris, $8 \mathrm{p}$.

Peyraud J.-L., Duhem K., 2013. « Les élevages laitiers de demain : exercice d'analyse prospective », INRA Productions Animales, vol. 26 (2), p. 221-230.

Pflimlin A., 2010. Europe laitière : valoriser tous les territoires pour construire l'avenir. Éditions France Agricole, 314 p.

You, 2015. « Contractualisation et modes de coordination dans la filière laitière », Économie Rurale, n³45, p. 87-100. 